\title{
A Case of Pancoast Tumor with Unusual Presentation
}

\author{
Bernadette Calabek ${ }^{1}$ Stefan Meng ${ }^{2}$ Sabine Pollanz ${ }^{1}$ Walter Klepetko ${ }^{3}$ Konrad Hoetzenecker ${ }^{3}$ \\ Felicitas Oberndorfer ${ }^{4}$ Wolfgang Grisold ${ }^{1}$
}

${ }^{1}$ Department of Neurology, Kaiser Franz Josef-Hospital, Ludwig Boltzmann-Institute of Neurooncology, Vienna, Austria

Address for correspondence Prof. Wolfgang Grisold, MD, Department

2 Department of Radiology, Kaiser Franz Josef-Hospital, Vienna, Austria of Neurology, Kaiser Franz Josef-Hospital, Ludwig Boltzmann-Institute

${ }^{3}$ Department of Thoracic Surgery, University Hospital of Vienna,

Vienna, Austria

${ }^{4}$ Clinical Institute of Pathology, University Hospital of Vienna,

Vienna, Austria

J Brachial Plex Peripher Nerve Inj 2015;10:e53-e56.

\begin{abstract}
Keywords

- brachial plexus

- lung cancer

- nerve infiltration

- outcome

- Pancoast tumor

- surgical resection

Introduction The Pancoast syndrome (PS) has been termed after Henry Pancoast. Its neurologic core symptoms include pain, radicular sensory and motor syndromes, and Horner syndrome. A PS is often the presenting sign of lung cancer and bears a grim prognosis.

Methods This case report describes an atypical onset of a lung tumor causing a PS. Electrophysiological examination was not conclusive. The diagnosis was confirmed by MRI, CT scan, and biopsy. The intervention consisted of preoperative chemo- and radiotherapy and was followed by an extensive surgical approach with histologically confirmed perineural invasion of the brachial plexus.

Results The postoperative period was dominated by neuropathic pain. Despite considerable loss of distal sensorimotor function of the right hand, the patient uses the extremity and has returned to professional life.

Discussion This observation triggered by the advances in general oncology and surgery also demonstrates the management of a lesion of the peripheral nervous system caused by cancer.
\end{abstract}

\section{Introduction}

The Pancoast syndrome (PS) has its name from an observation made by Henry Pancoast (1875-1939), and is often mentioned synonymously with lung cancer, although it can be associated with other neoplastic ${ }^{1,2}$ and non-neoplastic conditions. $^{3-8}$ Because of its location, the syndrome is also called superior sulcus tumor. Literature presents large case series, ${ }^{9}$ and also many single-case and small case series observations.

The PS in neurology is usually considered as composition of local pain, radicular sensorimotor symptoms, and Horner syndrome. Several "atypical presentations" with shoulder and neck pain, radiculopathy, ${ }^{10}$ elbow pain, and other focal signs ${ }^{11}$ have been described. Often the infiltration of the first rib, before invading the brachial plexus, can erroneously point to a local pain syndrome.

Prognostically the detection of a large lung tumor with infiltration of the brachial plexus is considered a dismal prognosis, and therapy is often restricted to supportive and palliative care, usually dominated by pain treatment. Follow-up studies of patients with PS show that distant metastasis also can often already be expected. ${ }^{12}$ This case demonstrates that intervention in a multidisciplinary setting followed by adequate pain therapy and rehabilitation received

August 3, 2014

accepted after revision

March 24, 2015

published online

May 2, 2015
DOI http://dx.doi.org/

10.1055/s-0035-1551654. ISSN $1749-7221$. (c) 2015 Georg Thieme Verlag KG
Stuttgart . New York

License terms

()ㅜ(1) $\circledast$ 
can be successful in regard to survival and also meaningful quality of life.

\section{Case Report}

A 45-year-old left-handed, nonsmoking woman came for neurologic evaluation, because of pain radiating into her right elbow and recently developing numbness in her fifth finger a few weeks prior to the consultation. As a magnetic resonance imaging (MRI) of the cervical spine suggested a spine disk protrusion at cervical segment $\mathrm{C} 8$, an orthopedic intervention had been planned.

She reported increasing progressive pain radiating into her right elbow for 9 months. Sensory loss in her fourth and fifth fingers and the ulnar side of the lower arm had evolved in the past weeks, resulting in clumsiness in her right hand. She was also unable to lie in supine position as this induced unbearable pain radiating into the right shoulder and trapezius muscle.

The neurologic investigation showed a right-sided Horner syndrome and a decreased triceps jerk. The right hand had a mild atrophy of the small hand muscles mimicking an ulnar nerve lesion with weakness of the interossei and lumbrical muscles. The sensory deficit was in $\mathrm{C} 8$ distribution. There was no anhidrosis. The palpation of Erb point was painful. Based on the clinical exam, a PS was suspected.

The patient was investigated with nerve conduction velocity (NCV) and electromyographic (EMG) studies, which were not conclusive. Motor and sensory NCVs of the right median and ulnar nerve were normal. The F-wave of the median nerve was normal, and the F-wave latency of the ulnar nerve was prolonged and showed dispersion. The sensory NCV of the right medial cutaneous antebrachii nerve was, however, absent (see - Fig. 2). EMG showed fibrillation potentials in the right abductor digiti quinti muscle, suggesting ongoing denervation, and confirmed the distribution of the lesion. The EMG of the abductor pollicis brevis muscle was normal.

Computed tomographic (CT) scan and MRI of the lung and brachial plexus revealed a large mass of the lung, infiltrating into the brachial plexus ( - Fig. 1A). Ultrasound showed a mass surrounding the brachial plexus (-Fig. 1B). A CT-guided biopsy revealed an adenocarcinoma of the lung. The patient was in a good condition and did not show any signs of metastatic spread. In interdisciplinary meetings it was decided to start chemotherapy (platinum/Navelbine) and focal radiotherapy (50 $\mathrm{Gy}$ ) before surgery.

Surgery was performed by a right-sided Paulson incision, which is the standard approach for posterior-type Pancoast tumors, ${ }^{13}$ and the thoracic cavity was entered through the second intercostal space. A standard upper lobe lobectomy was performed by ligating and dissecting the upper lobe vein, the upper lobe arteries, and by stapling the right upper lobe bronchus. Subsequently, laminectomies of Th1 to Th 4 and dissections of the corresponding intercostal nerve roots were performed. After this procedure, the extrathoracic part of the tumor was completely mobilized ( - Fig. 1C). The medial cord of the brachial plexus appeared infiltrated by the tumor and could not be spared to obtain clear resection margins. The
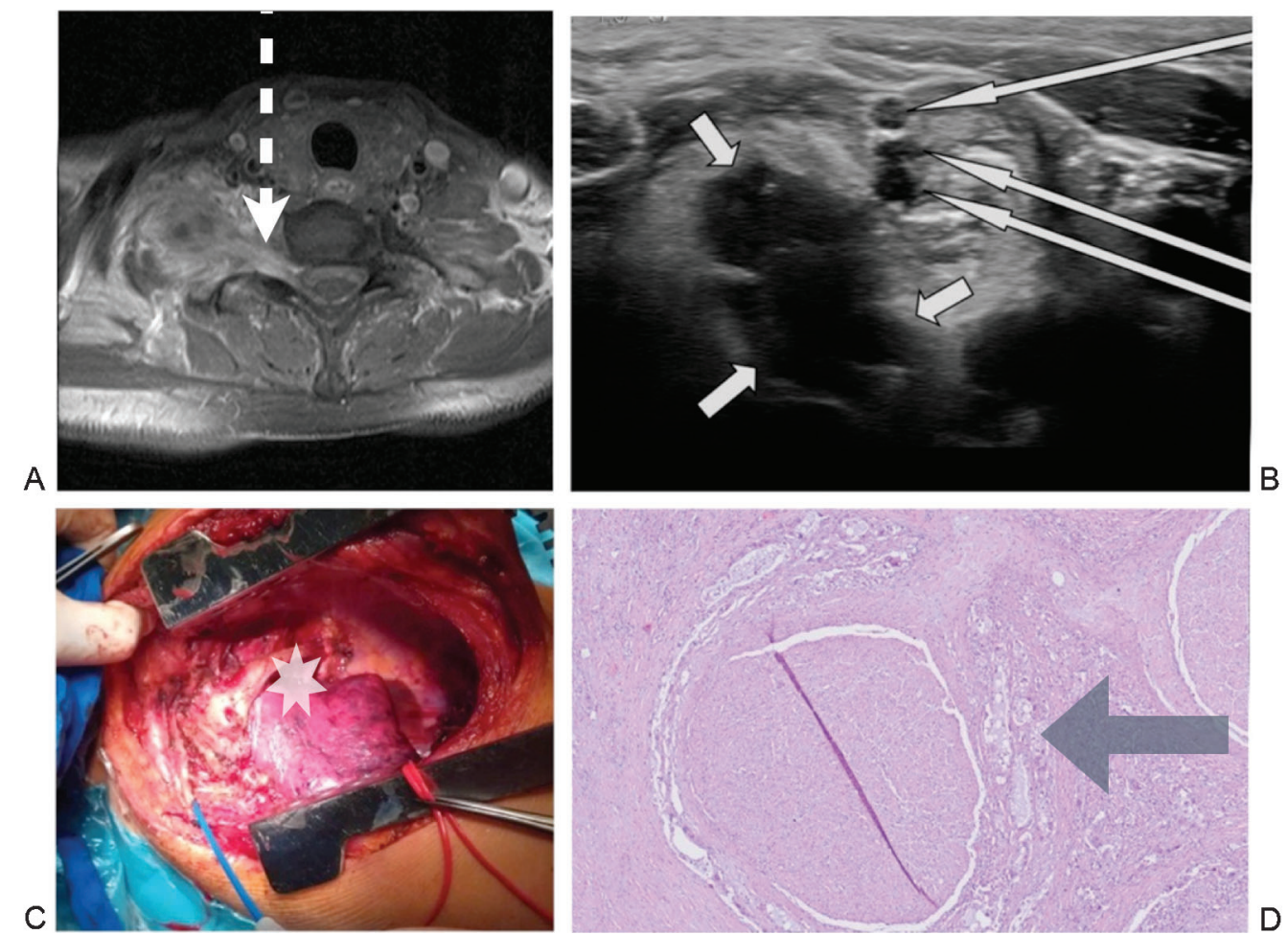

Fig. 1 (A) Magnetic resonance imaging of the brachial plexus axial T1 images showing axial lung tumor with infiltration of the brachial plexus (dotted arrow). (B) Ultrasound image of the brachial plexus (long arrows), tumor (short arrows). (C) Invasion of the superior thoracic aperture by tumor * The nerve fascicle was surrounded by tumor formations and infiltrated into the perineurium, but there was no intrafascicular spread. (D) Microscopic evaluation of the resection medial cord with adjacent tumor formations (arrow). 


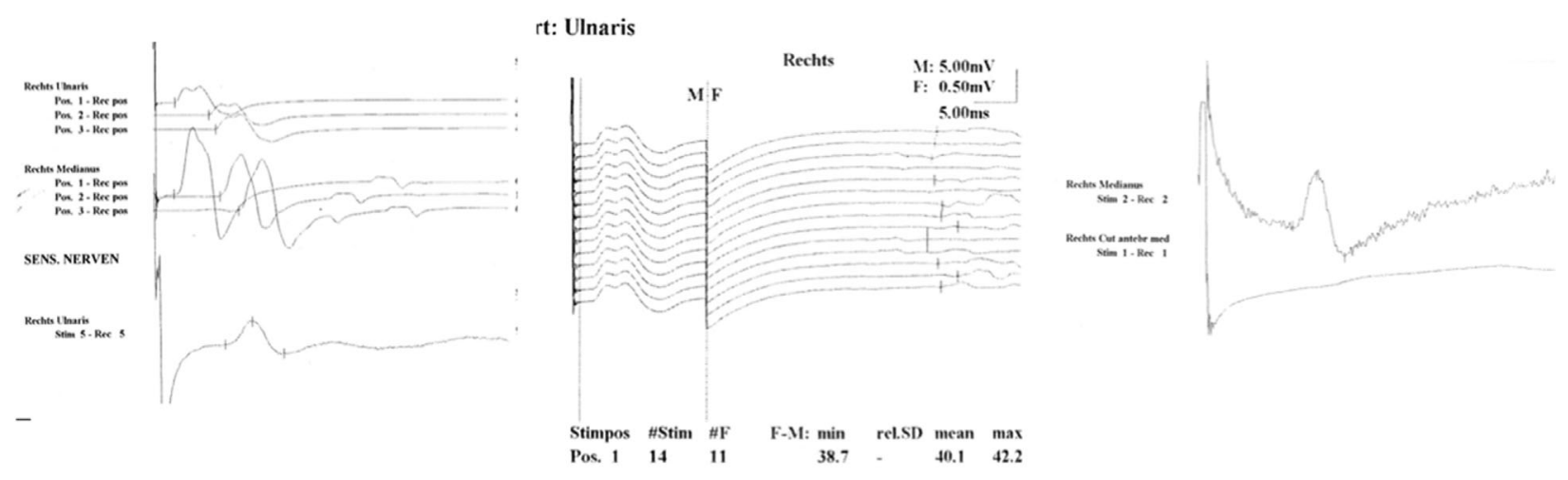

A

B

C

Fig. 2 Electrophysiology, preoperatively. (A) Motor conduction of median and ulnar nerve, sensory action potential of ulnar nerve preserved. (B) Ulnar nerve, delayed F-wave with dispersion. (C) Normal sensory conduction velocity of median nerve, absent cutaneous antebrachii medial nerve.

cord was surrounded proximal and distal by infiltration and transected by electrocautery. The in toto resected tumor tissue block could be finally removed, the subclavian vessels were reconstructed using ring-enforced polytetrafluoroethylene (PTFE) vascular grafts, and the thoracotomy was closed.

The histologic examination of the surgical specimen confirmed the biopsy-based diagnosis of an adenocarcinoma. The pathology report specified an invasive, moderately to poorly differentiated adenocarcinoma of the lung with $30 \%$ vital tumor cells, 65\% fibrosis, and 5\% necrosis after induction chemotherapy. The specimen of the brachial plexus showed abundant fibrotic stroma between carcinoma formations and a thick nerve bundle was identified. A nerve fascicle was partially surrounded by tumor formations and showed infiltration into the perineurium. There was no intrafascicular spread (-Fig. 1D).

The first follow-up 3 months postoperatively was characterized by severe neuropathic pain that could be managed with conventional therapy used for neuropathic pain. The intensity reduced over several months, and opiates could be terminated after 3 months. Therapy with gabapentin was maintained. Clinically a prominent winging of the right scapula, and preserved function of the $\mathrm{C} \% \mathrm{\%}$ muscles was noted. The muscles of the forearm and hand were paralyzed and the hand was held in a pseudoradial palsy fashion. The sensory perception of the hand was crude and did not allow the discrimination of specific qualities.

After 6 months the pain syndrome markedly improved. The neurologic findings remained identical. Because of focused physiotherapy, she was using her affected hand and lower arm hand as a counter bearing to execute hand movements with her left hand.

\section{Discussion}

This case shows symptoms, signs, findings, and treatment in a patient with PS syndrome. The course of the disease had several unusual aspects as the onset of pain radiating into the elbow 9 months previously and a spread into the right shoulder. The sensory symptoms in the eighth cervical segment appeared late. A concomitant Horner syndrome had appeared several months earlier, but was not recognized as such.

Clinically PS can present with uncharacteristic signs such as joint pain, ${ }^{11,14}$ which causes undue delays in the diagnosis. $^{15}$

Also in this case, the electrophysiologic studies were misleading. The NCV studies showed normal motor and sensor conductions of the median and ulnar nerve, the medial cutaneous antebrachial nerve NCV was missing, and also the F-wave of the ulnar nerve was prolonged and dispersed (-Fig. 2). The neurogenic changes in the small hand (C8/Th1) muscles confirmed an acute ongoing denervation. The definite diagnosis was made by CT scan and MRI. Also, the ultrasound of the brachial plexus demonstrated the plexus surrounded by tumor mass. A CT scan-guided biopsy finally confirmed the diagnosis of adenocarcinoma.

Presurgically the patient received local radio- and chemotherapy, and finally underwent lobectomy with partial resection of the brachial plexus. Invasion and destruction of surrounding tissue by a Pancoast tumor including the brachial plexus has often been considered as a palliative and incurable situation. In this case, the patient had no evidence of generalized metastases and was in a younger age group and in a good general medical condition. A combination of thoracic, plastic, and reconstructive surgery has been reported successful in some cases ${ }^{13,16-20}$ however, despite combined approaches, survival remains poor. ${ }^{21}$ The histologic examination showed a thick nerve bundle of the brachial plexus embedded in abundant fibrotic stroma between carcinoma formations. Besides, small carcinoma formations surround the fascicle and infiltrate into the perineurium.

Recently, Davis and Knight published their series of Pancoast tumors in which resection of the brachial plexus could be avoided by neurolysis performed by a dedicated neurosurgeon. ${ }^{16}$ Posterior-type Pancoast tumors, which have a contact/infiltration of the neurologic structures, are routinely operated together with a neurosurgeon in our institution. In the described case neurolysis was considered impossible. The tumor had broad contact to the medial cord and an 
infiltration of the cord could not be excluded. Therefore, the medial cord was sacrificed to obtain clear resection margins. This procedure was preoperatively discussed with the patient and she consented to all resulting sequelae.

Infiltration of peripheral nerves occurs in the cerebrospinal fluid space, skin tumors, and often in ENT (ear-nosethroat) tumors. Infiltration of the nerve plexus occurs also in the brachial and in particular the sacral plexus and rarely in other peripheral nerves except in some types of hematologic diseases as lymphoma ${ }^{1}$ and leukemia. The type and pattern of peripheral nerve infiltration have been classified ${ }^{22}$ into "true nerve metastasis" within the nerve, direct invasion, invasion along the nerve sheath, pushing and stretching and engulfing. In this case, the spread of the tumor was engulfing as well as metastatic along the vessels. Generally, spread of malignant cells in peripheral nerves is most frequent in hematologic conditions, rare in cancer, and even rare in sarcoma. In general pathology autonomic nerve invasion is observed in several tumors as pancreatic and prostate cancer and is a bad prognostic sign.

The follow-up was primarily associated with severe neuropathic pain, which required a treatment combination of gabapentin, amitriptyline, and opioids, and subsided within several months to a controlled level. The postoperative status showed a good function of the shoulder muscles, with the exception of a marked scapular winging. The hand function showed a severe sensory and motor loss, with only a slight perception of touch and marked trophic changes. She is able to use her upper limb by movements of the shoulder and upper arm muscles and as a counter bearing for objects when using her left hand. Despite this impairment, she has continued her office job 5 months after the operation. Three years after the surgery, the patient is without any evidence of tumor recurrence. The neuropathic pain has considerably improved.

In addition to the uncharacteristic appearance of the PS, this case demonstrates a smooth transition from clinical findings, imaging, histopathologic findings, and postoperative development, and demonstrates that also in a PS with perineural invasion meaningful positive results can be obtained.

\section{Consent}

The patient was informed that data obtained would be submitted for publication, and written consent was obtained from the patient.

\section{References}

1 Mills PR, Han LY, Dick R, Clarke SW. Pancoast syndrome caused by a high grade B cell lymphoma. Thorax 1994;49(1):92-93
2 Bosnjak R, Bacovnik U, Podnar S, Benedicic M. T1-nerve root neuroma presenting with apical mass and Horner's syndrome. J Brachial Plex Peripher Nerve Inj 2007;2:7

3 Ambrosic F. [A case of pleural echinococcal cyst with PancoastTobias syndrome]. Srp Arh Celok Lek 1962;90:753-757

4 Arenas Gordillo M, Ortega Ruiz F, Otero Candelera R, Caballero Oliver A, Blanco Orozco A, Calderón Osuna E. [Pancoast syndrome caused by lung tuberculosis]. Arch Bronconeumol 1998;34(5): 266-268

5 Bansal M, Martin SR, Rudnicki SA, Hiatt KM, Mireles-Cabodevila E. A rapidly progressing Pancoast syndrome due to pulmonary mucormycosis: a case report. J Med Case Reports 2011;5:388

6 Fibla JJ, Penagos JC, León C. [Pseudo-Pancoast syndrome caused by a solitary fibrous tumor of the pleura]. Arch Bronconeumol 2004; 40(5):244-245

7 Hirtenstein J. Pancoast syndrome due to acute osteomyelitis. BMJ 1956;2(4993):645

8 Reuss-Borst MA, Becker V, Sattler B, Grupp C, Müller GA. Clinical images: Wegener's granulomatosis presenting as Pancoast tumor. Arthritis Rheum 2000;43(2):467

9 Yukiue H, Tanahashi M, Haneda H, Suzuki E, Yoshii N, Niwa H. [Surgical treatment for Pancoast tumors-significance of surgical approach and induction chemoradiotherapy]. Kyobu Geka 2010; 63(1):4-8

10 Vargo MM, Flood KM. Pancoast tumor presenting as cervical radiculopathy. Arch Phys Med Rehabil 1990;71(8):606-609

11 Deng PB, Luo YY, Hu CP, Zhou LH. [Misdiagnosis of Pancoast cancer: analysis of 26 cases]. Zhonghua Jie He He Hu Xi Za Zhi 2011;34(9): 663-665

12 Shah H, Anker CJ, Bogart J, Graziano S, Shah CM. Brain: the common site of relapse in patients with Pancoast or superior sulcus tumors. J Thorac Oncol 2006;1(9):1020-1022

13 Pitz CC, de la Rivière AB, van Swieten HA, Duurkens VA, Lammers JW, van den Bosch JM. Surgical treatment of Pancoast tumours. Eur J Cardiothorac Surg 2004;26(1):202-208

14 Kovach SG, Huslig EL. Shoulder pain and Pancoast tumor: a diagnostic dilemma. J Manipulative Physiol Ther 1984;7(1):25-31

15 Ichinohe K, Takahashi M, Tooyama N. Delay by patients and doctors in treatment of Pancoast tumor. Wien Klin Wochenschr 2006;118(13-14):405-410

16 Davis GA, Knight S. Pancoast tumor resection with preservation of brachial plexus and hand function. Neurosurg Focus 2007;22(6): E15

17 Shahian DM, Neptune WB, Ellis FH Jr. Pancoast tumors: improved survival with preoperative and postoperative radiotherapy. Ann Thorac Surg 1987;43(1):32-38

18 Remmen HJ, Lacquet LK, Van Son JA, Morshuis WJ, Cox AL. Surgical treatment of Pancoast tumor. J Cardiovasc Surg (Torino) 1993; 34(2):157-161

19 Okubo K, Wada H, Fukuse T, et al. Treatment of Pancoast tumors. Combined irradiation and radical resection. Thorac Cardiovasc Surg 1995;43(5):284-286

20 Muscolino G, Valente M, Andreani S. Pancoast tumours: clinical assessment and long-term results of combined radiosurgical treatment. Thorax 1997;52(3):284-286

21 Kodama K, Okami J, Maeda J, et al. [Complete resection of Pancoast tumor following induction chemoradiotherapy improves survival]. Kyobu Geka 2010;63(1):9-15

22 Meller I, Alkalay D, Mozes M, Geffen DB, Ferit T. Isolated metastases to peripheral nerves. Report of five cases involving the brachial plexus. Cancer 1995;76(10):1829-1832 